\title{
La calidez en enfermería: formulación de constructo y variables relacionadas ${ }^{1}$
}

\author{
Zita Elena Lagos Sánchez² \\ Cibele Andrucioli de Mattos-Pimenta ${ }^{3}$ \\ María Teresa Urrutia ${ }^{4}$
}

doi:10.11144/Javeriana.ie18-1.cefc

Cómo citar: Lagos Sánchez ZE, Mattos-Pimenta CA de, Urrutia MT. Calidez en enfermería: formulación de constructo y variables relacionadas. Investig Enferm. Imagen Desarr. 2016;18(1): 95-113. http://dx.doi.org/10.11144/Javeriana.ie18-1.cefc

1. Artículo derivado de investigación. Recibido: 24 de diciembre de 2014. Aprobado: 17 de septiembre de 2015.

2. Doctora (c) en Enfermeria, PROESA. DINTER, Escuela de Enfermeria, Universidad de São Paulo, Brasil. Correo electrónico: zita.lagos@gmail.com

3. Doctora en Enfermería, Universidade de São Paulo, Brasil. Correo electrónico: parpca@ usp.br

4. Magíster en Nutrición, Mención en Salud Pública, Pontificia Universidad Católica de Chile (PUC), Chile. Correo electrónico: murrutis@uc.cl 


\section{Resumen}

La calidez es un fenómeno subjetivo fundamental en las relaciones personales. La calidez se contagia y, así, puede ser un factor favorecedor en el cuidado, mas no existe consenso en su definición ni dominios. Medir la calidez es significativo, pues es un predictor de satisfacción usuaria; sin embargo, no se encontraron modos de medirla, lo que motivó esta revisión. Objetivos: Identificar en la revisión teórica comportamientos que representen la calidez del enfermero y operar el constructo calidez en enfermería y variables relacionadas. Método: Revisión integrativa que abarcó la exploración y análisis de la evidencia sobre calidez, la cual corresponde a la primera etapa del proyecto de investigación titulado: Construcción y validación de una escala de medición de la calidez presente en los enfermeros, donde se pretende construir y validar una escala que permita medir este fenómeno. Se revisaron definiciones de calidez en diccionarios, textos y artículos de diversas bases de datos. Los descriptores utilizados fueron: calidez, calidez y enfermería, en español e inglés. Para definir el constructo y sus variables se complementó el modelo de tres fases de Luiz Pasquali con los procedimientos de Ethel Bauzer y Floyd Fowler. Resultados: Se formuló el constructo calidez en enfermería, se identificaron 2 dominios, 28 variables verbales, 9 no verbales y 93 comportamientos. Conclusiones: Los resultados de la fase teórica del modelo aplicado permitieron formular constructo, dominios y variables de calidez en enfermería. Estos aportes son de interés para la gestión del cuidado enfermero y enseñanza, pues se relacionan directamente con la satisfacción usuaria.

Palabras clave: cuidado de enfermeria; escalas; satisfacción del paciente

\section{The Warmth in Nursing: Construct Formulation and Related Variables}

\section{Abstract}

The warmth is a subjective phenomenon, fundamental in human relationships. The warmth is contagious, so may be a factor favoring care, but there is no consensus on its definition and domains. Since warmth is a predictor of user satisfaction, it is relevant to use an appropriate way to measure it; however, there have not been found ways to measure it, which motivated this revision. The three-stage procedure of Luiz Pasquali was used to do that, which considers Theoretical, Empirical and Analytical phases. This paper presents the results of the first theoretical phase: behaviors associated with warmth previously identified in the literature. Objectives: To identify behaviors that represent the warmth of the Nurse by reviewing specialized literature, and to operationalize the construct warmth and related variables. Method: Integrative literature review that included the exploration and analysis of the evidence on warmth, which corresponds to the first stage of the research project entitled: Construction and validation of a scale for measuring this warmth in Nursing, which aims to build and validate a scale to measure this phenomenon. This work corresponds to the Theoretical Phase of Pascuali's model. Definition of warmth was revised by using dictionaries and technical articles from various repositories. The descriptors used were "warmth" and "warmth and nursing" in Spanish and English. To define the construct and its variables, the Ethel Bauzer's procedures and Floyd Fowler's method were also considered to supplement the Pascuali's model. Results: Formulation of the construct Warmth in Nursing which includes, 2 domains, 28 verbal variables, 9 nonverbal variables and 93 behaviors. Conclusions: The results of the theoretical phase of the applied model, allowed to formulate the construct domains and variables of Warmth in Nursing. These contributions are of interest for the management of nursing care and teaching, as are directly related to user satisfaction.

Keywords: nursing care; scales; patient satisfaction 


\section{O calor humano em enfermagem: formulação do construto e variáveis relacionadas}

\section{Resumo}

O calor humano é um fenômeno subjetivo fundamental nos relacionamentos. O calor humano é contagioso, por isso pode ser um fator relevante no cuidado, mas não há consenso sobre sua definição ou domínios. Medir o calor humano é relevante porque é um preditor de satisfação do usuário, no entanto, não encontraram maneiras de medir, o que motivou essa revisão. Foi usado o modelo de três fases do autor brasileiro Luiz Pasquali: teórica, empírica e analítica. Este trabalho mostra os resultados da primeira fase teórica: a literatura identifica comportamentos que são associados com o calor humano. Objetivos: Identificar numa fase de revisão teórica, os comportamentos que representam o calor humano do enfermeiro. Operacionalizar a definição do construto calor humano em enfermagem, bem como identificar as variáveis. Método: Revisão integrativa que abrange a exploração e análise das evidências em calidez (calor humano), o que corresponde à primeira fase do projeto de pesquisa intitulado: Construção e validação de uma escala para medir esse calor em Enfermagem, que tem como objetivo construir e validar uma escala para medir este fenómeno. Este trabalho corresponde ao resultado da fase teórico do modelo. Os descritores utilizados foram calidez, calidez dos enfermeiros e sua correspondente em inglês e espanhol. Para a definição de calor humano e construção de variáveis que a representam, o modelo de três fases de Luiz Pasquali, foi complementado por procedimentos de Ethel Bauzer e Floyd Fowler. Resultados: Foi formulado o construto Calor humano do Enfermeiro, dois domínios, 28 variáveis verbais e 9 não-verbais. 93 comportamentos totais identificados com a calidez dos enfermeiros. Conclusões: Os resultados da fase teórica do modelo aplicado permitiram formular os domínios construir e variáveis do calidez em enfermagem. Estas contribuições são de interesse para a gestão da assistência de enfermagem e ensino, como estão diretamente relacionados à satisfação do usuário.

Palauras chave: cuidado de enfermagem; escalas; a satisfação do paciente 


\section{Introducción}

La calidez es un fenómeno subjetivo, una emoción primitiva y fundamental (1). Sujeta de contagio fluye entre una persona y otra (2). Ante una persona enferma o vulnerable, este flujo puede ser un factor que favorezca el ánimo y la recuperación del paciente. A su vez, la entrega de cuidados provoca en el receptor sensaciones y emociones que se asocian con el grado de satisfacción (3). Por este motivo, en la literatura que analiza temas de calidad o satisfacción usuaria se reitera la importancia de la calidez (4).

Ser cálido parece obvio en el momento de cuidar; sin embargo, no existe un modelo teórico de calidez en enfermería, lo que dificulta su gestión. De los referenciales teóricos analizados se seleccionaron aquellos que tratan comportamientos asociables con calidez, resumidos a continuación:

- Modelo de humanización en salud, de Juan Carlos Bermejo, 2003 (5): no conceptúa calidez, mas destaca comportamientos que se pueden relacionar: "La cordialidad, la amabilidad, la cercanía, y la empatía". Según el autor, estas habilidades "son educables ya que una persona puede aprender a ser cordial".

- $\quad$ Modelo de habilidades sociales de Vicente Caballo, 1993 (6): no define calidez; sin embargo, describe comportamientos: "Hacer y aceptar cumplidos, expresar amor, agrado y afecto, iniciar y mantener conversaciones, gesticular".

- Modelo de calidez terapéutica de C. Truax, 1971 (7): aborda la calidez y la empatía. "La calidez, así como la empatía, es considerada una variable fundamental de la alianza terapéutica"; no conceptúa la calidez.

- Modelo de calidez y competencia de Cuddy y Fiske, 2012 (8): la calidez se relaciona con competencia. "Una persona cálida es percibida como competente".

- $\quad$ Filosofía de humanitud, de Y. Gineste y R. Marescotti, 1987 (9): no define calidez; pero incluye comportamientos: "La verticalidad, el contacto ocular, el modo de conversar, la capacidad de tocar el otro y sonreír" (10). Mario Simões, en el artículo "Cuidar em humanitude" menciona otros comportamientos: “Tocar, mirar a los ojos, llamar por el nombre, usar un tono de voz suave, firme y melodioso" (11).

Los instrumentos hallados para medir la calidez lo hacen unidos a otros fenómenos, por ejemplo: Truax Relationship Questionnaire (TRQ), que evalúa calidez y empatía (12); The Warmth and Permissiveness Scales, que mide calidez y permisividad (13), y el CPI, Male and Female Warmth Scales for the California Psychological Inventory, que mide calidez y género (14). No se encontraron instrumentos que midan la calidez en enfermería. Esto es relevante, debido a que las acciones del cuidado enfermero están presentes en un alto porcentaje de las intervenciones hospitalarias. De este modo, si 
estás acciones se ejecutan con un componente de cuidado cálido, pueden influir en la medición de la satisfacción usuaria.

La búsqueda bibliográfica no mostró estudios sobre gestión ni sistematización de la calidez. Los artículos que conceptúan calidez no describen sus dimensiones, lo que dificulta desarrollar este concepto y su práctica. E1 objetivo de este artículo es presentar la formulación del constructo calidez, sus dominios y los comportamientos que lo caracterizan, derivados de la fase teórica del modelo de construcción de escalas de Pasquali (15).

\section{Método}

Se llevó a cabo una revisión integrativa para definir el constructo calidez y las variables que la representan. Se adaptó la metodología sugerida por el autor Luiz Pasquali, con los pasos recomendados por la autora Ethel Bauzer (16) y los procedimientos descritos por Floyd Fowler (17). Pasquali sugiere una metodología de tres fases (18): teórica, empírica y analítica. Ethlel Bauzer propone el procedimiento para construir un instrumento. Floyd Fowler describe recomendaciones para medir fenómenos subjetivos. En este trabajo se presentan los resultados de la primera fase (teórica), que corresponden a la exploración de la teoría para identificar comportamientos que se asocien a calidez. Con esto se formuló el constructo y se operacionalizaron las variables.

La búsqueda se efectuó en bases del área de salud, enfermería y psicología. Las bases de datos revisadas fueron: Bireme, Science Direct, American Association for the Advancement of Science (AAAS), Link Springer, Al Dia, Web of Science, Web of Science, Biblioteca Cochrane Iberoamericana y Cochrane Plus, EBSCO CINAHL Plus y CUIDEN. No se limitó en años; hubo bases que no presentaron resultados (Aldia(C), WOS(C), Biblioteca Cochrane(C). Los criterios de búsqueda fueron: artículos en español, inglés o portugués y los descriptores utilizados: warmth y warmth and nursing y calidez en enfermeria. Ninguno de ellos normalizados en los Descriptores de Ciencias de la Salud (DeSC). Los criterios de inclusión en la selección fueron estudios relacionados con calidez en enfermería o calidez en la relación interpersonal. El criterio de exclusión se dio para aquellos estudios que trataban la calidez como calor local. Con las palabras calidez y enfermería (nursing and warmth) se encontraron artículos en su mayoría relacionados con la satisfacción usuaria. El instrumento utilizado para análisis de rigor de los artículos fueron las Guias Caspe (19-21).

\section{Resultados}

En la primera búsqueda se obtuvieron 448 artículos, de los cuales se seleccionaron 37 que cumplieron con los criterios de inclusión: 20 en idioma inglés (54\%), 13 en español (35\%) y 4 en portugués (11\%, 3 de Portugal y 1 de Brasil). En la tabla 1 se presenta la sintesis de los artículos seleccionados y los hallazgos relacionados. 


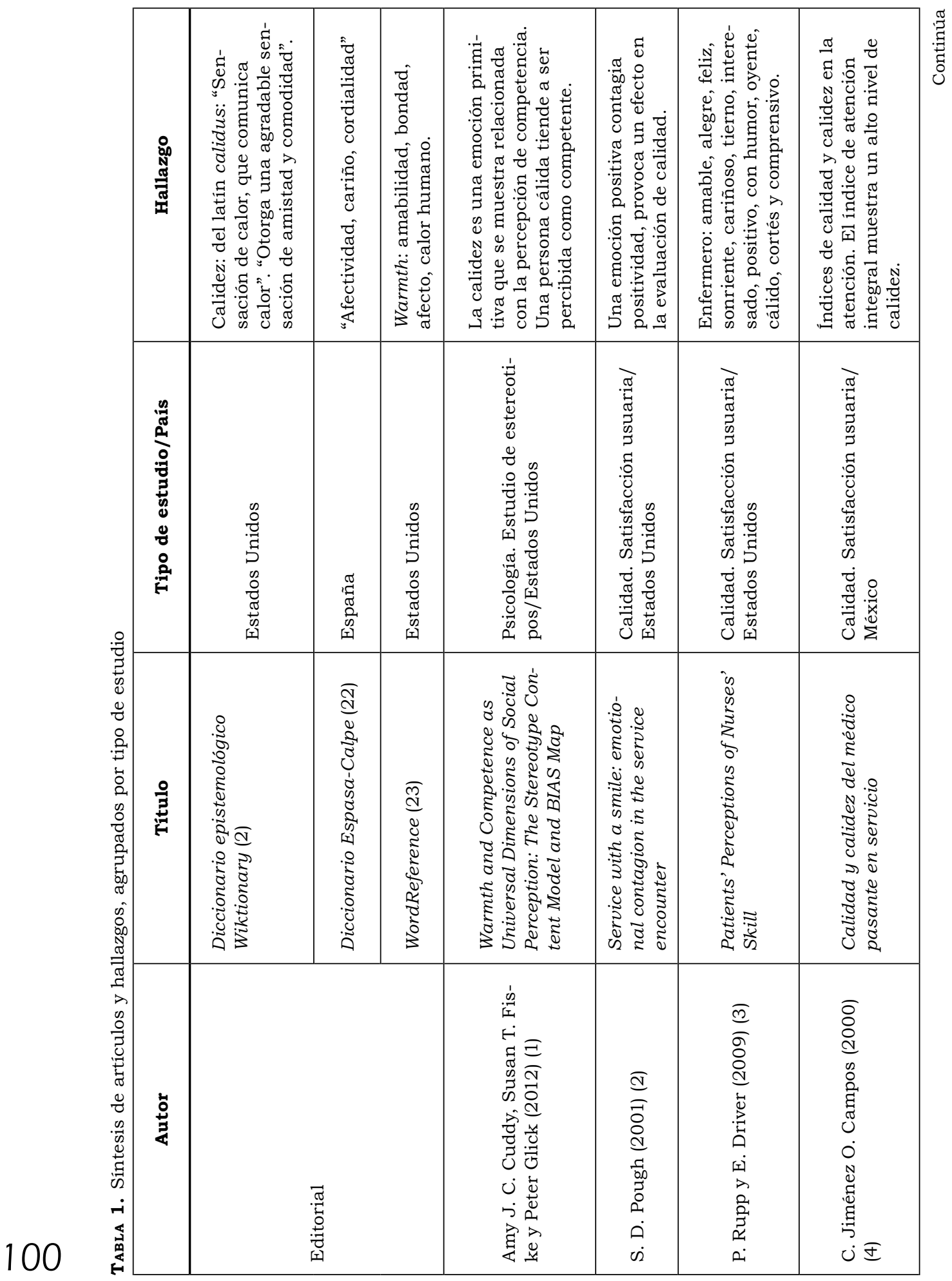




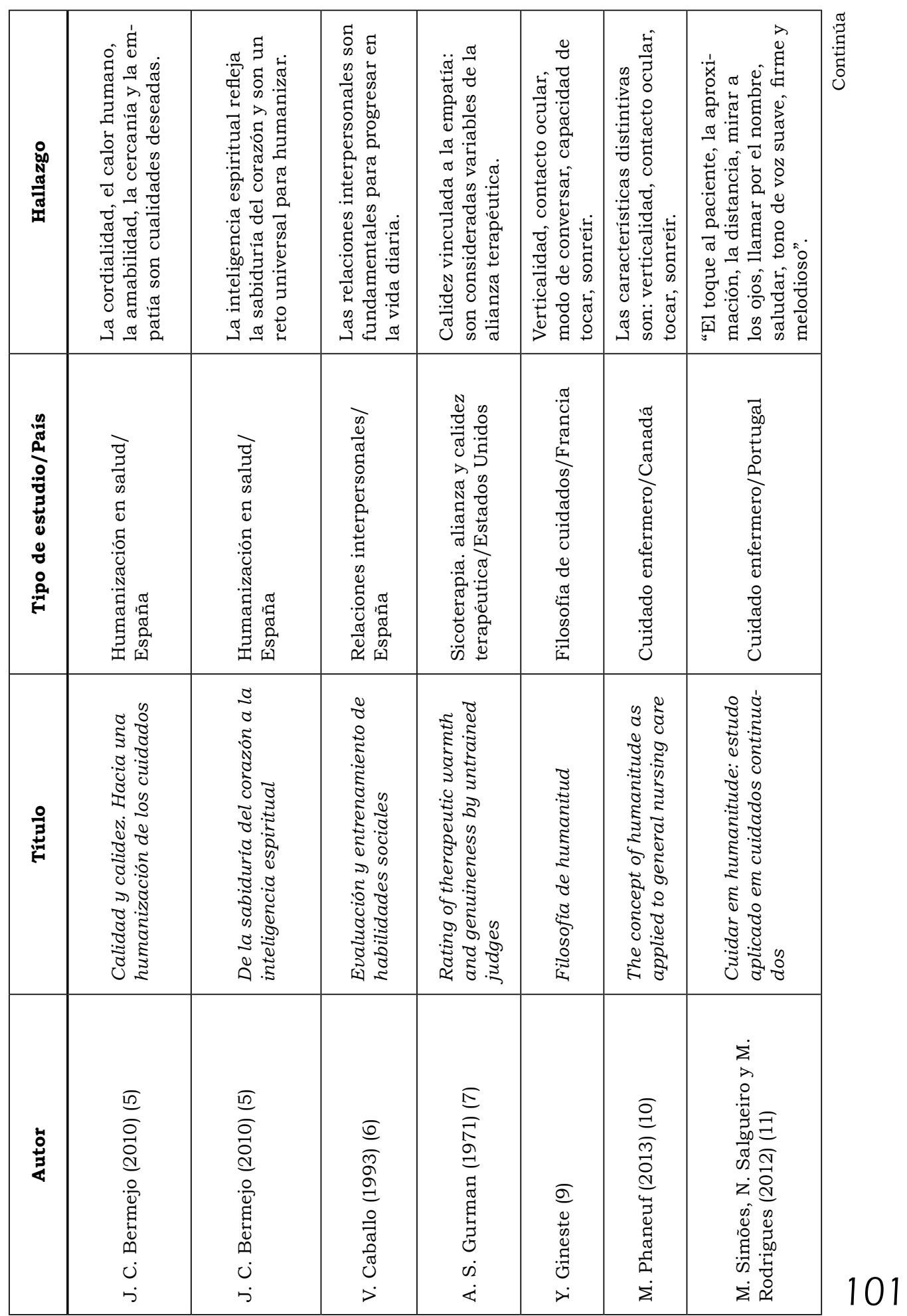




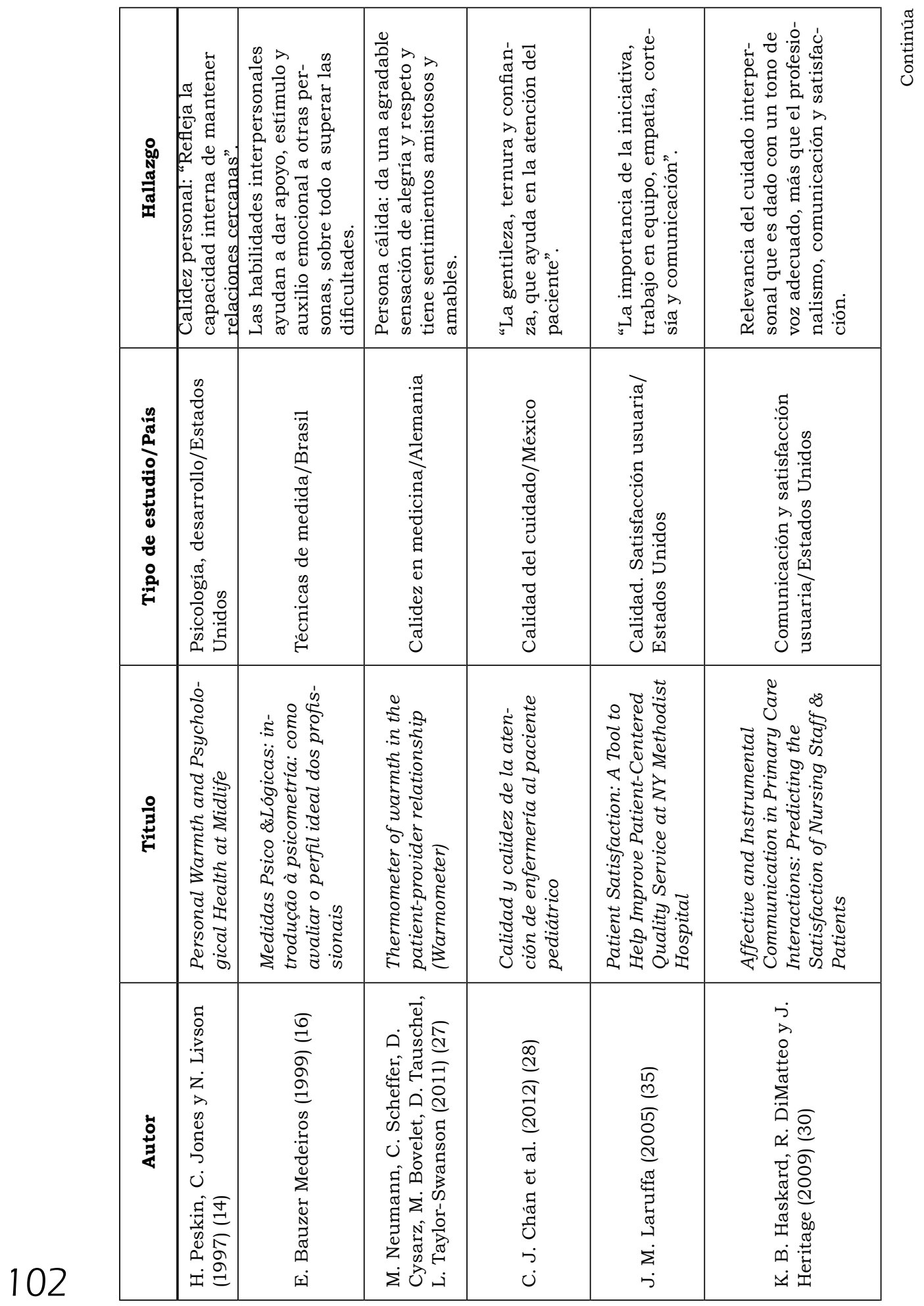




\begin{tabular}{|c|c|c|c|c|c|c|}
\hline 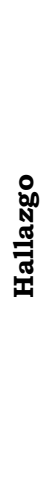 & 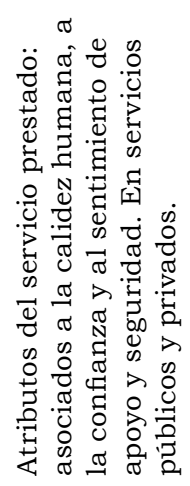 & 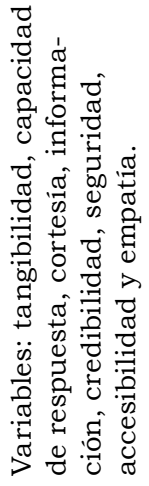 & 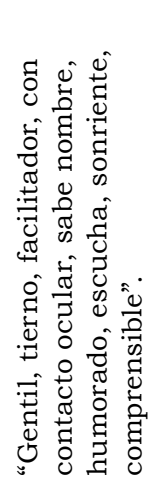 & 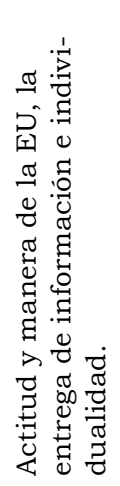 & 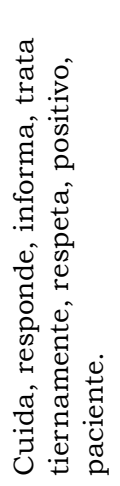 & 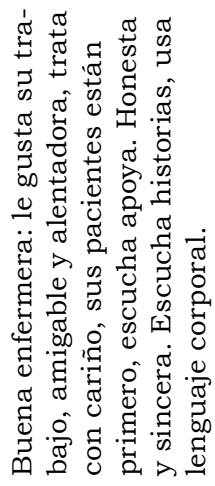 \\
\hline 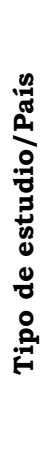 & 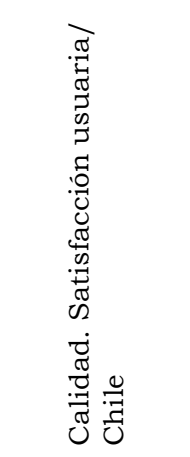 & $\begin{array}{l}0 \\
0 \\
0 \\
\pi \\
0 \\
0 \\
\text { I1 } \\
0 \\
0 \\
0 \\
0 \\
0 \\
0 \\
0 \\
0 \\
0 \\
0 \\
0 \\
0 \\
0 \\
0 \\
0 \\
0 \\
0 \\
0\end{array}$ & 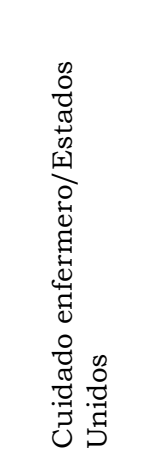 & 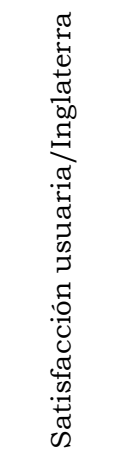 & 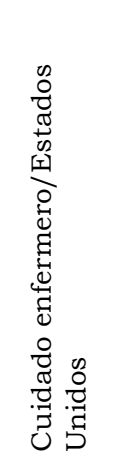 & 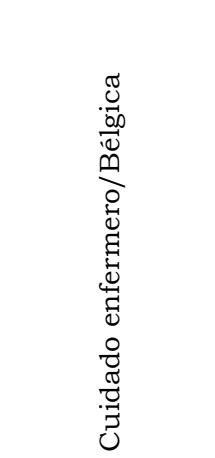 \\
\hline 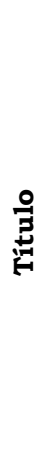 & 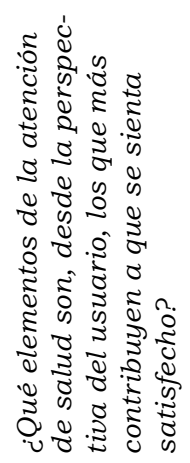 & 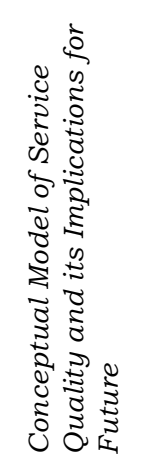 & 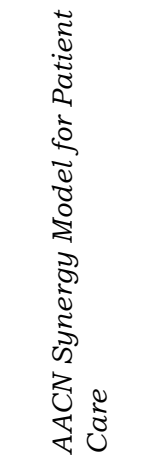 & 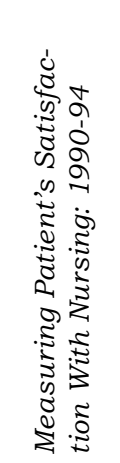 & 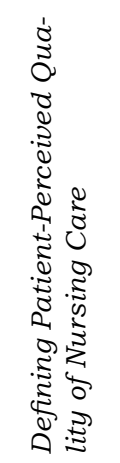 & 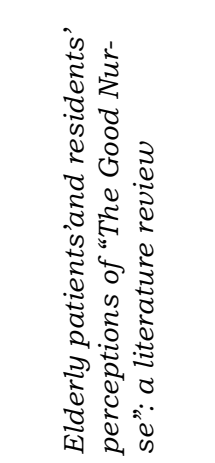 \\
\hline 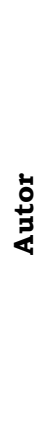 & 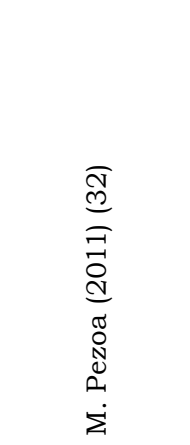 & 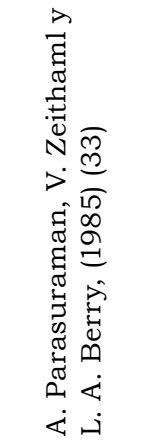 & 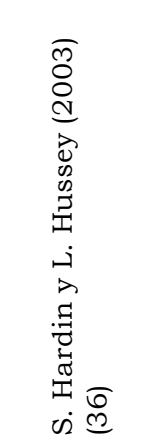 & 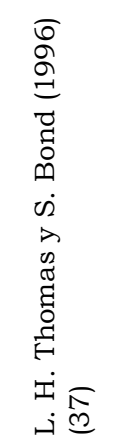 & 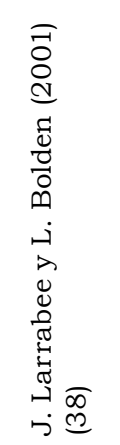 & 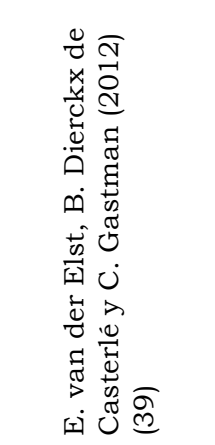 \\
\hline
\end{tabular}

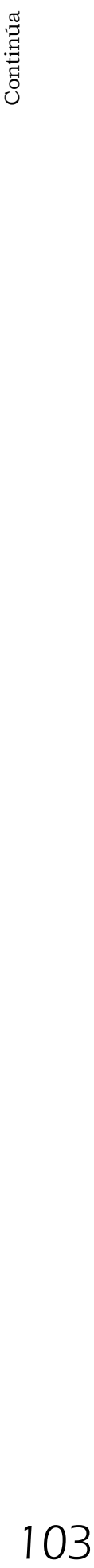




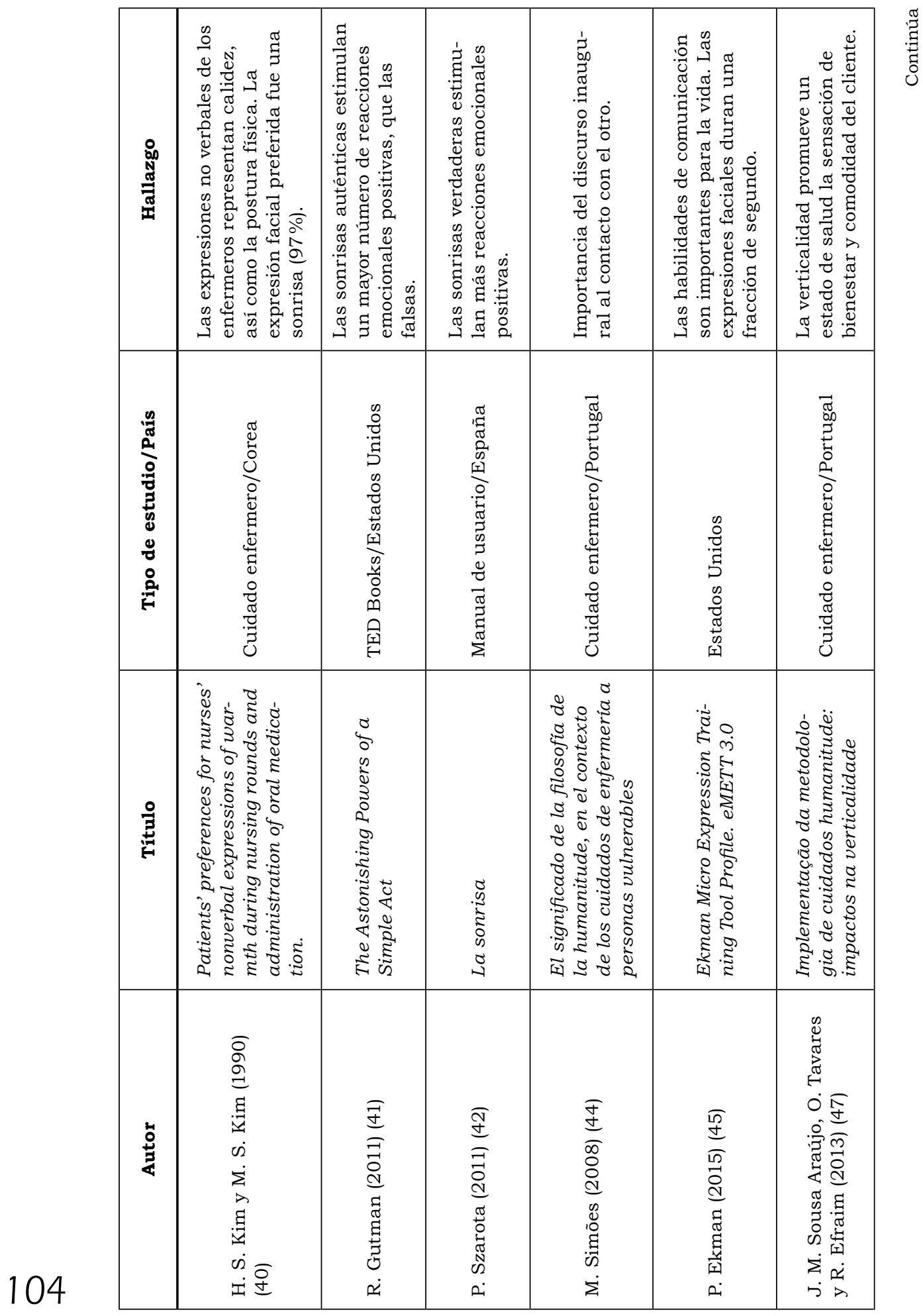




\begin{tabular}{|c|c|c|c|c|c|}
\hline 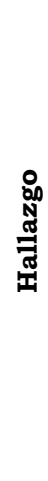 & 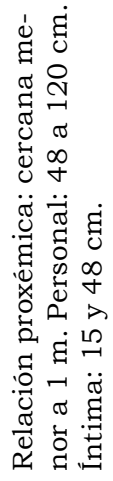 & 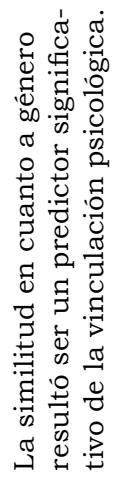 & 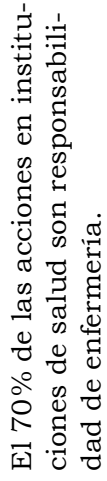 & 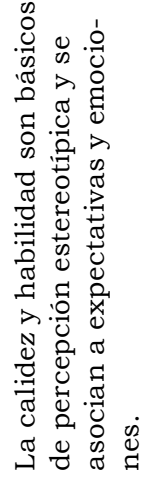 & 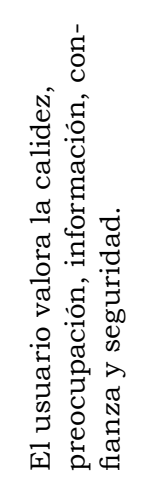 \\
\hline 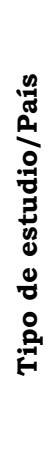 & 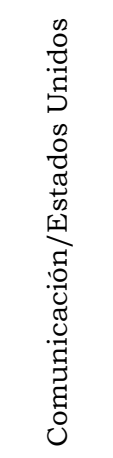 & 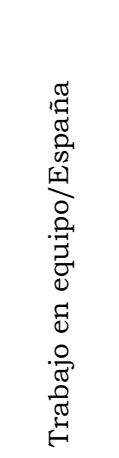 & 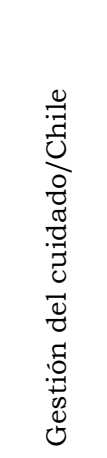 & 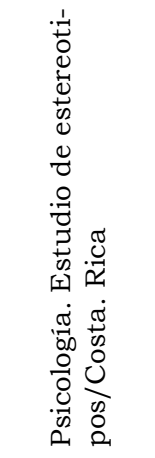 & 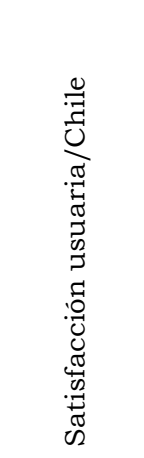 \\
\hline 号 & 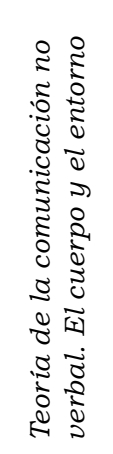 & 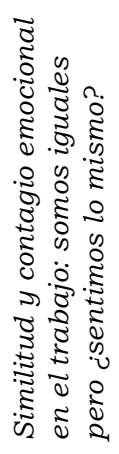 & 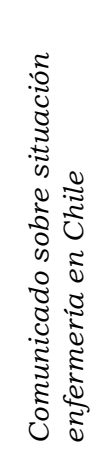 & 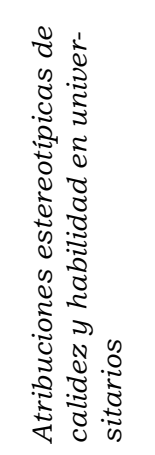 & 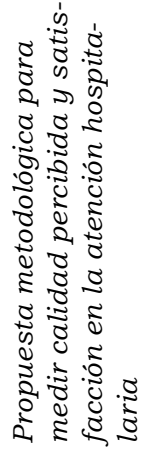 \\
\hline 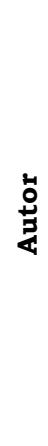 & 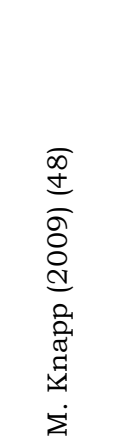 & 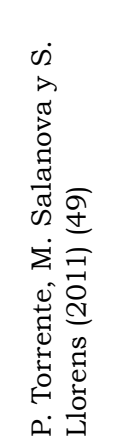 & 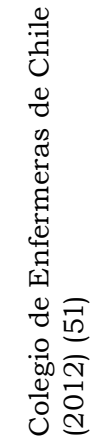 & 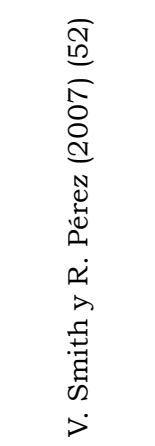 & 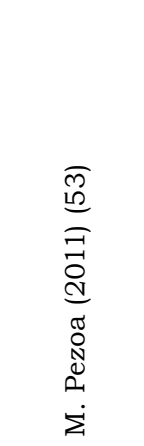 \\
\hline
\end{tabular}


Con estos hallazgos y siguiendo los procedimientos recomendados por el modelo de Pasquali, se procedió a formular el constructo de calidez y posteriormente a determinar comportamientos relacionados.

\section{Formulación de constructo y variables asociadas a calidez, derivadas de fase teórica del modelo adaptado de Pasquali}

Definición de calidez. Se inició con la búsqueda en diccionarios y en artículos de satisfacción usuaria y calidad que contenian atributos interpretables como expresiones de la calidez: amistad, cariño, cordialidad, amabilidad, bondad, afecto y calor humano, incorporadas a la definición operacional.

Calidez, del latín calidus, que significa: "Sensación de calor o que comunica calor, proporciona una agradable sensación de amistad y comodidad", sinónimo "acogedor" (20). El Diccionario de la lengua española lo define como "Calor o ardor" (21), y no representa el atributo buscado. El Diccionario Espasa-Calpe dice: "Afectividad, cariño, cordialidad" (22). En inglés warmth tiene una connotación interpersonal: "Amabilidad, bondad y afecto: calor humano" (23). En portugués se define como "Calor o ardor" y no representa al atributo (24). De las autoras Cuddy y Fiske (25): "Es una emoción primitiva para determinar si las intenciones del otro son positivas o negativas". Del artículo de Peskin, Jones y Livson (26) se incorporó el atributo: "Refleja la capacidad interna de mantener relaciones cercanas".

De Neumann y colaboradores (27) se incorporó a la definición: "Provoca sentimientos agradables de alegría, amistad, ternura y amabilidad". De los autores Chán y colaboradores (28) se utilizó el siguiente aspecto de la definición de calidez: "La gentileza, ternura y confianza, que ayuda en la atención del paciente". Con estos aportes se realizó la definición operacional de calidez en enfermería.

Definición operacional. "La capacidad de establecer y mantener una relación cercana, de acogida, que demuestre por medio de comportamientos verbales y no verbales: ternura, confianza, afecto, cariño y calor humano; de manera que para el otro signifique una experiencia agradable".

Determinación y definición de las variables asociadas a la calidez. En adelante se presentan los comportamientos asociados a la calidez que permitieron determinar las variables asociadas:

- Cuddy, Fiske y Glick: "Calidez es una emoción".

- Pough: "Una emoción positiva contagia positividad, repercute en la calidad".

- Rupp: ser "Amable, alegre, feliz, sonriente, cariñoso, tierno, interesado, confidente, positivo, con humor, organizado, sin prisa, oyente, establece lazos".

- Press Ganey® (29): tener "Iniciativa, empatía, cortesía y comunicación".

- Haskard, DiMatteo y Heritage (30): "Tono de voz adecuado".

- Modelo de sinergia de la AACN: enfermero muy competente. "Aquellos tiernos, alegres, cálidos, corteses, y comprensivos” (31). 
- Pezoa, SIS MINSAL, Chile (32): “Atributos asociados a la calidez humana, a la confianza y al sentimiento de apoyo, que tanto en servicios públicos y privados, marcan una diferencia en el nivel de satisfacción".

- Parasuraman (33): "Cortesía, información, credibilidad, accesibilidad y empatía".

- Jiménez y Campos (34): “Comunicación, confianza, respeto, amabilidad y orientación al paciente y familia, seguridad y respeto por las costumbres".

- Laruffa (35): "Habilidades de comunicación del equipo de enfermería".

- Hardin y Hussey (36): “Gentil, tierno, facilitador, interesado, con contacto ocular, conoce el nombre, con humor, atento, escucha, es intuitivo, amigable, alegre, sonriente, feliz, actitud positiva, lenguaje comprensible".

- Thomas (37): "Entrega información y mantiene individualidad".

- Larrabee (38): "Cuida, responde preguntas, da información exacta, trata tiernamente, respeta, actitud positiva y paciente".

- Van der Elst Dierckx de Casterle y Gastmans (39): "Amigable y alentador, trata con cariño, los pacientes están primero, escucha, apoya, es honesto, próximo y recíproco. Escucha las historias de los pacientes. Tiene comunicación abierta y usa el lenguaje corporal. Trata a la familia y amigos amigablemente".

- $\quad$ Kim y Kim (40): “El contacto visual y la expresión facial preferida por los pacientes es la sonrisa. La distancia es importante".

- Gutman (41): "Las sonrisas auténticas estimulan un mayor número de reacciones emocionales positivas".

- Szarota (42): "Sonrisas sentidas estimulan más reacciones positivas".

- Pough (43): "Los clientes captan el afecto de los empleados a través del contagio emocional. El flujo de emoción positiva está relacionada con la calidad".

Operacionalización de las variables que representan la calidez. Con la incorporación de los atributos mencionados se pudieron establecer dos agrupaciones o dominios asociados con la calidez: comportamientos verbales y no verbales. Estos se reagruparon en 28 variables verbales y 9 no verbales, representadas por 93 comportamientos de calidez del enfermero.

Comportamientos verbales. Se identificaron 28:

1. Saludar: discurso inaugural individual al contacto con el otro (44).

2. Presentarse: menciona el nombre y cargo al contacto con el usuario.

3. Discurso: da orientaciones sobre eventos o rutinas importantes.

4. Tono de voz: usa un tono suave, firme y melodioso (45-53).

5. Responder dudas: responde a las consultas del interlocutor.

6. Respetar: considera los valores y costumbres del otro.

7. Tiempo de respuesta: es prudente, no deja esperando por una respuesta.

8. Amabilidad: tiene delicadeza en el trato.

9. Alegría: demuestra un sentimiento de apariencia placentero.

10. Amistad: demuestra confianza y sentimiento desinteresado. 
11. Atención: demuestra cortesía, respeto, cuidado en lo que se va a decir.

12. Afecto: demuestra cariño y simpatía hacia el otro.

13. Bondad: es amable, demuestra inclinación hacia lo considerado bueno.

14. Auxilio emocional: atento para ayudar a expresar y escuchar sentimientos.

15. Conversación: inicia y mantiene por un tiempo razonable, con lenguaje claro.

16. Cumplidos: hace y acepta cumplidos con expresión de agrado y afecto.

17. Escuchar: tiene actitud de atención y respuesta.

18. Compromiso: cumple las promesas.

19. Credibilidad: su discurso es aceptado como verdadero.

20. Cordialidad: es amable y afectuoso, controla los sentimientos negativos.

21. Cariño: demuestra amor o afecto hacia el otro.

22. Confianza: provoca un sentimiento de seguridad.

23. Accesibilidad: provoca un sentimiento de poder llegar a su persona.

24. Interés: demuestra inclinación hacia la otra persona.

25. Ser positivo: transmite un mensaje realista, optimista y con sentido del humor.

26. Seguridad: transmite conocimientos y competencias en su actuar.

27. Expresión de sentimientos: manifiesta lo que siente al momento de interactuar.

28. Ternura: provoca sentimientos de simpatía y dulzura.

Comportamientos no verbales. Se identificaron 9:

1. Sonreir: arquea los labios hacia arriba.

2. Contacto visual: orienta el rostro y la mirada hacia el paciente (46).

3. Gesticular: acompaña el discurso con las manos y movimientos corporales.

4. Tocar: contacta con las manos, con sentido de apoyo o ternura.

5. Verticalidad: se coloca a la altura del otro (47).

6. Postura: postura ligeramente inclinada.

7. Proxemia: distancia entre dos personas, menor a un metro (48).

8. Contagio emocional: provoca, demuestra y contagia sentimientos (49).

9. Sigilo: actúa con cuidado de no molestar o meter ruido (50).

\section{Discusión}

Estas variables son de interés para la gestión del cuidado enfermero y la enseñanza, pues se relacionan directamente con la satisfacción del usuario. La operacionalización de la calidez y sus variables tiene implicaciones en la práctica clínica de enfermería, aun cuando puede ser usada en otros estamentos con otros fines. En Chile sería de utilidad en los estudios de calidad, puesto que, según el Colegio de Enfermeras, el $70 \%$ de las acciones que se realizan en una institución de salud son de responsabilidad de enfermería (51); de esta forma, contar con estas variables permitiria generar un indicador de calidez altamente correlacionado con la satisfacción usuaria. 
A su vez, tener una definición de calidez en enfermería permite aunar el criterio y profundizar en un fenómeno poco estudiado. La identificación de categorias y definiciones operacionales serán las bases para construir una escala que permita medir la calidez presente en los enfermeros. Contar con un indicador de calidez puede convertirse en un predictor exitoso de satisfacción. Con esta definición y relación de comportamientos, los enfermeros analizan cuáles son aquellos que ya utilizan en la asistencia de los usuarios, y cuáles incorporar. También los docentes pueden analizar qué contenidos sobre calidez en enfermería se están enseñando y reflexionar cuáles es posible incorporar a la gestión del cuidado cálido. Al atraer interés en el tema, se moviliza la incorporación del conocimiento en la capacitación del personal, lo que puede fortalecer las politicas institucionales asociadas al cuidado. Por último, de acuerdo con Neumann, la calidez se considera "el rasgo más poderoso de la personalidad" (27); en consecuencia, trabajar con calidez generaría ambientes cálidos que están en directa relación con el bienestar del paciente.

\section{Conclusiones}

Finalizada la revisión de la literatura, se respondió al objetivo planteado en la fase teórica del modelo presentado: se formuló la definición de calidez en enfermería y se identificaron 2 dominios, 37 variables $(28$ verbales y 9 comportamientos no verbales) que permitirán a futuro medir la calidez. No existe evidencia sobre medición de la calidez de los enfermeros. Los artículos que estudian la medición de la calidez la abordan como fenómeno compartido con otras dimensiones; el único artículo que la mide aisladamente está enfocado en médicos.

La definición de calidez es compleja, dado que en los diccionarios en español y portugués no tiene una connotación relacional; sin embargo, esta condición sí se da en inglés. A su vez, la descripción de las variables asociadas a calidez forma parte de un trabajo teórico de relación e interpretación de la evidencia seleccionada, pues son pocos los estudios que mencionan variables directamente incorporadas al fenómeno. Entre ellos está el trabajo de las autoras Cuddy, Fiske y Glick; el estudio de Pezoa, de Chile; las escalas que miden calidez asociada con otras variables, y el trabajo de Smith y Pérez.

La formulación del constructo calidez, los dominios y comportamientos identificados son la base para construir una escala de calidez - actualmente en desarrollo por las autoras - que será el primer instrumento válido para medir la calidez en enfermería. A su vez, aportará a la evidencia e investigación y permitirá avanzar en este referencial teórico de calidez, gestión del cuidado cálido y calidad de la atención de enfermería.

\section{Conflictos de interés}

Las autoras declaran no tener conflictos de interés en este proyecto. 


\section{Financiación}

Fondos locales de apoyo a la investigación.

\section{Referencias}

1. Cuddy AJC, Fiske ST, Glick P. Warmth and competence as universal dimensions of social perception: the stereotype content model and the bias map. Advances in Experimental Social Psychology [internet]. 2008;(40):61-149. Disponible en: http://www.hbs.edu/faculty/Pages/ item.aspx?num $=34511$

2. Pough SD. Service with a smile: emotional contagion in the service encounter. Acad Manage J [Internet]. 2001;44(5):1018-27. Disponible en: http://www.communicationcache.com/uploads/1/0/8/8/10887248/ service_with_a_smile_and_encounter_satisfaction-_emotional_contagion_and_appraisal_mechanisms.pdf

3. Rupp P, Driver E. Patients' perceptions of nurses' skill. Crit Care Nurse [internet]. 2009;29(4):24-7. Disponible en: http://goo.gl/Bhrg5X

4. Jiménez C, Campos O. Calidad y calidez del médico pasante en servicio. Salud Problema [internet]. 2000 [citado sep 2012];5(9):24-30. Disponible en: http://goo.gl/AJL3zE

5. Bermejo JC. Calidad y calidez: hacia una humanización de los cuidados [internet]. [Citado 11 de abril de 2013]. Disponible en: http:// goo.gl/QUtGbD

6. Caballo V. Manual de evaluación y entrenamiento de las habilidades sociales. Madrid: Siglo XXI; 1993.

7. Truax C. The empirical emphasis in psychotherapy: A symposium. Effective ingredients in psychotherapy: An approach to unraveling the patient-therapist interaction. J Counsel Psychol. 1963;10(3):25663. doi:10.1037/h0041061.

8. Cuddy AJC, Fiske ST, Glick P. Universal dimensions of social cognition: warmth and competence. Trends in Cognitive Sciences [Internet]. 2006 [citado 8 ago 2012];11(2):3-7. Disponible en: http://psych.colorado.edu/wiki/lib/exe/fetch.php?media=labs:social-brownbag:fiske_ cuddy_glick_tics.pdf.

9. Gineste Y, Marescotti R. Filosofía de humanitud [internet]. [Citado 10 jun 2013]. Disponible en: http://goo.gl/KvrvOb.

10. Phaneuf $\mathrm{M}$. The concept of humanitude as applied to general nursing care [Internet]. [Citado 1 sep 2013];1-20. Disponible en: http:// goo.gl/rgnrFh.

11. Simões M, Salgueiro N, Rodrigues M. Cuidar em humanitude: estudo aplicado em cuidados continuados. Rev Enf Ref [Internet]. 2012 [citado 12 sep 2013];IIIa Série(6):81-93. Disponible en: http://goo.gl/DEjzy8.

12. Truax C. Rating of therapeutic warmth and genuineness by untrained judges. Psychological Reports [Internet]. 1971 [citado 22 sep 2013];28:711-14. Disponible en: ttp://www.amsciepub.com/doi/ pdf/10.2466/pr0.1971.28.3.711.

13. Christensen CM. Relationships between pupil achievement, pupil affect-need, teacher warmth, and teacher permissiveness. J Educ 
Psychol [Internet]. 1960 [citado 22 sep 2013];51(3):169-72. Disponible en: http://dx.doi.org/10.1037/h0044666

14. Peskin H, Jones C, Livson N. Personal warmth and psychological health at midlife. J Adult Dev [Internet]. 1997 [citado 20 oct 2013];4(2):73. Disponible en: http://link.springer.com/article/10.1007/BF02510082

15. Pasquali L. Teoría dos testes na psicología e na educação. 4 a ed. Petrópolis: Vozes; 2003.

16. Bauzer ME. Medidas Psico \& Lógicas: introdução à psicometría. Como avaliar o perfil ideal dos profissionais. Rio do Janeiro: Ediouro; 1999.

17. Floyd JF. Improving survey questions: design and evaluation (applied social research methods). Vol. 38. Thousand Oaks, CA: Sage; 1991.

18. Pasquali L. Psicometría. Rev Esc Enferm USP [Internet]. 2009 [citado 3 jul 2013];43(Esp):992-9. Disponible en: http://www.scielo.br/pdf/ reeusp/v43nspe/a02v43ns.pdf.

19. Guías Caspe. Programa de lectura crítica CASPe: entendiendo la evidencia sobre la eficacia clínica [internet]. [Citado 20 ene 2013]. Disponible en: http://www.redcaspe.org/.

20. Diccionario epistemológico Wiktionary [Internet] [citado 1 nov 2013]. Disponible en: http://es.wiktionary.org/wiki/c\%C3\%A1lido.

21. Real Academia Española. Diccionario de la lengua española [internet]; 2014. [Citado 16 abril 2013]. Disponible en: http://www.rae.es.

22. WordReference [internet]. [Citado 16 abr 2013]. Disponible en: http:// www.wordreference.com/definicion/calidez

23. The Free Dictionary [Internet]. [Citado 16 abr 2013]. Disponible en: http://www.thefreedictionary.com.

24. Diccionario portugués on line [Internet]. [Citado 16 abr 2013]. Disponible en: http://www.wordreference.com/definicion/calidez.

25. Cuddy AJC, Glick P, Beninger A. The dynamics of warmth and competence judgments, and their outcomes in organizations. Res Organ Behav [Internet]. 2011 [citado 25 ago 2012];31:65. Disponible en: http:// www.hbs.edu/faculty/Pages/item.aspx?num $=41451$.

26. Peskin H, Jones C, Livson N. Personal warmth and psychological health at midlife. J Adult Dev [Internet]. 1997 [citado 3 ago 2013];4(2):73. Disponible en http://link.springer.com/article/10.1007/BF02510082

27. Neumann M, Scheffer C, Cysarz D, Bovelet M, Tauschel D, TaylorSwanson L, et al. Thermometer of warmth in the patient-provider relationship (Warmometer)--theory-based development of a patient self-report measure and initial validation using cognitive interview methodology. Patient Educ Couns [Internet]. 2011 [citado 10 may 2013];82(3):361-9. Disponible en: http://goo.gl/p60Yhp.

28. Chán CJ, Gómez SL, Manrique C, Serralde SH, Ortiz R. Calidad y calidez de la atención de enfermería al paciente pediátrico, una investigación mixta. Paraninfo digital monográficos de investigación en salud [Internet]. 2012 [citado 1 oct 2013];(16):3. Disponible en: http:// goo.gl/zgPmuE 
29. Press Ganey knowledge summary: patient satisfaction with emotional and spiritual care [citado marzo 2012]. Disponible en: http://hmablogs.hma.com/hmachaplains/files/2010/05/Press-Ganey-PatientSatisfaction.pdf

30. Haskard KB, M. DiMatteo R, Heritage J. affective and instrumental communication in primary care interactions: predicting the satisfaction of nursing staff and patients. Health Commun [Internet]. 2009 [citado abril de 2013];24(1):21-32. Disponible en: http://www.researchgate.net/ publication/23995276_Affective_and_instrumental_communication_ in_primary_care_interactions_predicting_the_satisfaction_of_nursing staff_and_patients

31. AACN Certification Corporation. The AACN synergy model for patient care. American Association of Critical-Care Nurses [Internet]. 2003 [citado nov 2012]. Disponible en: http://goo.gl/vaFssq

32. Pezoa M. ¿Qué elementos de la atención de salud son, desde la perspectiva del usuario los que más contribuyen a que se sienta satisfecho en la atención hospitalaria? [Internet]. Santiago: Superintendencia de Salud, Gobierno de Chile; 2011 [citado 13 abr 2012]. Disponible en: http://www.superdesalud.gob.cl

33. Parasuraman A, Zeithaml V, Berry L. A conceptual model of service quality and its implications for future. Research Journal of Marketing [Internet]. 1985 [citado 12 may 2013]; 49(4): 41-50. Disponible en: http://areas.kenan-flagler.unc.edu/Marketing/FacultyStaff/zeitha$\mathrm{ml} /$ Selected \%20Publications/A\%20Conceptual\%20Model\%20of\%20 Service \%20Quality \%20and \%20Its \%20Implications \%20for \%20 Future\%20Research.pdf.

34. González G. Calidad y calidez en atención y servicio al cliente. B\&M News. Seminarios [Internet]. 2013 [citado sep 2012]. Disponible en: http://www.businessinbajio.com/revista/calidad-calidez-en-atencion-servicio-al-cliente/

35. Laruffa JB. Patient satisfaction: a tool to help improve patient-centered quality service at New York Methodist Hospital [internet]. Capstone Project, Binghampton University; 2005 [citado mar 2012]. Disponible en: http://goo.gl/Ucyyqt.

36. Hardin S, Hussey L. AACN synergy model for patient care: case study of a CHF patient. Crit Care Nurse [Internet]. 2003 [citado oct 2012];23(1):75. Disponible en: http://goo.gl/Yg0FDm.

37. Bond S, Thomas LH. Measuring patient's satisfaction with nursing: 1990-1994. J Adv Nurs [Internet]. 1996 [citado jul 2012];23:747-56. Disponible en: http://www.ncbi.nlm.nih.gov/pubmed/1537991.

38. Larrabee J, Bolden L. Definig patient-perceived quality of nursing care. J Nurs Care Qual [Internet]. 2001 [citado mar 2012];16(1):3460. Disponible en: http://www.ncbi.nlm.nih.gov/pubmed/11668855.

39. Van der Elst E, Dierckx de Casterlé B, Gastman C. Elderly patients and residents' perceptions of "The Good Nurse": a literature review. J Med Ethics [Internet]. 2012 [citado marzo de 2012];38:93-7. Disponible en: http://goo.gl/dUkolX 
40. Kim HS, Kim MS. Patients' preferences for nurses' nonverbal expressions of warmth during nursing rounds and administration of oral medication. Kanho Hakhoe Chi. [Internet]. 1990 [citado 10 may 2012];20(3):381-98. Disponible en: http://goo.gl/OBLa1N.

41. Gutman R. Smile: The astonishing powers of a simple act. New York: Forbes; 2011. Capítulo 17.

42. Szarota P. La sonrisa. Madrid: Editorial UOC; 2011.

43. Pough SD. Driving service effectiveness through employee-customer linkage. Acad Manage Exec [Internet]. 2002 [citado abril 2013];16(4):7980. Disponible en: http://www.researchgate.net/publication/254465586_ Driving_service_effectiveness_through_employee-customer_linkage

44. Simões M, Rodrigues M, Willow N. El significado de la filosofía de la humanitude en el contexto de los cuidados de enfermería a personas dependientes y vulnerables. Revista Referencia [Internet]. 2008 [citado sep 2014];II. ${ }^{a}$ Série(7):97-105. Disponible en: http://www.humanitude.pt/content/1409607129_artigo_de_revisa_o1_pdf.pdf.

45. Paul Ekman Group. Ekman Micro Expression Training Tool Profile. eMETT 3.0 [internet] [citado 29 nov 2013]. Disponible en: http://www. paulekman.com.

46. Humanitude IGM France. La méthodologie des soins Gineste-Marescotti IGM [internet]; 2010. [Citado 10 jul 2013]. Disponible en: http://goo. gl/30fvQs.

47. Sousa Araújo JM, Tavares O, Efraim R, Salgueiro N. Implementação da metodologia de cuidados humanitude: impactos na verticalidade. Enfermagem de Saúde do Idoso [Internet]. [Citado 13 jun 2013]. Disponible en: http://goo.gl/hDTWmj.

48. Knapp M. Teoría de la comunicación no verbal: el cuerpo y el entorno. México: Paidós Mexicana; 2009.

49. Torrente P, Salanova M, Llorens S. Similitud y contagio emocional en el trabajo: somos iguales pero... ¿sentimos lo mismo? Fòrum de Recerca [Internet]. 2011 [citado 20 nov 2013];16:827-36. Disponible en: http://goo.gl/Jk1kpH.

50. Real Academia Española. Diccionario de la lengua española: sigilo [internet]. 2001 [citado 10 nov 2013]. Disponible en: http://www.rae.es

51. Colegio de Enfermeras de Chile. Comunicado del 26 de noviembre del 2012 [internet]. [Citado 10 may 2013]. Disponible en: http:// www.achieen.cl/uploads/documentos/Comunicado\%20CONJUNTO. pdf0.36318100\%201354026766762191007.pdf.

52. Smith V, Pérez R. Atribuciones estereotípicas de calidez y habilidad en jóvenes universitarios. Rev Ciencias Sociales [Internet]. 2007 [citado jun 2014];(III-IV):117-8: 129-147. Disponible en: http://revistas. ucr.ac.cr/index.php/sociales/article/viewFile/11019/10399.

53. Pezoa M. Propuesta metodológica para medir calidad percibida y satisfacción en la atención de salud hospitalaria [Internet]. Santiago de Chile: Departamento de Estudios y Desarrollo, Supersalud y Gobierno de Chile; 2011 [citado abr 2012]. Disponible en: http://www.supersalud.gob.cl/documentacion/569/articles-7317_recurso_1.pdf. 